https://doi.org/10.15407/ujpe63.2.174

A.YA. DZYUBLIK, V.I. SLISENKO, V.V. MYKHAYLOVSKYY

Institute for Nuclear Research, Nat. Acad. of Sci. of Ukraine

(47, Props. Narky, Kyiv 03680,Ukraine; e-mail: dzyublik@ukr.net)

\title{
SYMMETRIC LAUE DIFFRACTION OF SPHERICAL NEUTRON WAVES IN ABSORBING CRYSTALS
}

\section{Introduction}

The diffraction of x-rays, Mössbauer or synchrotron radiation, and neutrons is widely used in numerous studies of the crystal structure and other applications. Well-known are the works of Kagan and Afanas'ev, who developed the dynamical scattering theory of Mössbauer rays [1] and neutrons [2]. They predicted the suppression effect for reactions and inelastic channels during the diffraction in perfect crystals, which was completely confirmed experimentally. In particular, the suppression of the $(n, \gamma)$ reaction has been observed by Shilshtein et al. [3, 4] in experiments on the neutron diffraction in a CdS crystal with the neutron energy close to the resonant energy $E_{0}=0.178 \mathrm{eV}$ of ${ }^{113} \mathrm{Cd}$. This suppression effect is similar to the effect of anomalous absorption of x-rays in crystals (Borrmann effect) [5-8].

In $[1,2]$, the electromagnetic waves and neutrons were described by plane waves. At the same time, in typical experiments on the Laue diffraction, the in-

(c) A.YA. DZYUBLIK, V.I. SLISENKO, V.V. MYKHAYLOVSKYY, 2018

174 cident waves first pass a narrow slit and only afterward penetrate the crystal (see Fig. 1). In this case, the incident waves are characterized by some aperture and should be described already by a wave packet. In addition, both the transmitting and diffracted waves inside the crystal travel within the region confined by a triangle called Borrmann's triangle [6-8]. Kato [9-12] developed a theory for such diffraction in the approximation of spherical waves, by assuming that the angular spreading of incident x-ray waves exceeds significantly the small diffraction interval of the order of several seconds of arc. The distribution of the diffracted beam intensity along the base of the Borrmann triangle manifests a fringe structure caused by the interference of two waves, transmitting inside the crystal with different wave vectors. Just their interference provides the familiar Pendellösung oscillations of the intensity [5-8]. Shull [13-15] used the Kato's theory to interpret the results of his subtle experiments on the Laue diffraction of neutrons in perfect crystals of silicon and germanium. Measuring the fringe patterns of the diffracted neutron beam, he determined, with high precision, the coherent scattering lengths of

ISSN 2071-0194. Ukr. J. Phys. 2018. Vol. 63, No. 2 
neutrons by the nuclei of silicon [13] and germanium [15]. It is worth noting also works $[16,17]$ concerned with the Laue diffraction of neutrons at the Bragg angle close to $\pi / 2$, which open new perspectives in the neutron optics.

In Kato's theory, the integrals over the glancing angle, which describe the x-ray wave packet inside the crystal, are estimated with the aid of the stationaryphase method (see also [7]) applicable only in the case of extremely weakly absorbing crystals. Here, we study the symmetric Laue diffraction of spherical neutron waves in crystals with nuclei having lowlying resonant levels (e.g., ${ }^{113} \mathrm{Cd}$ ). In this case of strongly absorbing crystals, we have done estimations by means of the saddle-point method, being more general than the stationary-phase one. Some peculiarities of the symmetric Laue diffraction of spherical Mössbauer waves in perfect crystals were already analyzed in [18]. Both coherent Rayleigh and resonant scatterings of $\gamma$-quanta by Mössbauer nuclei have been taken there into consideration.

\section{Basic Formulas}

Let the incident neutron as $t \rightarrow-\infty$ be described by the initial wave packet

$\Psi_{\text {in }}(\mathbf{r}, t)=\int d \boldsymbol{\kappa} f(\boldsymbol{\kappa}) e^{i \boldsymbol{\kappa} \mathbf{r}-i E t / \hbar}$,

where the wave vector $\kappa$, energy $E=\hbar^{2} \kappa^{2} / 2 m$, and $m$ is the neutron mass. For brevity, we omit the spin factor, which is not changed in the coherent scattering by a nonmagnetic crystal with unpolarized nuclei.

We choose the coordinate frame $x, y, z$ having the origin on the face crystal surface in the middle of the collimating slit with the axis $z$ perpendicular to the crystal surface and directed inside the crystal and the axis $y$ along the slit. We consider here the symmetric Laue diffraction, when the axis $z$ is parallel to the reflecting planes. We suppose that all the incident neutrons propagate along the plane $x, z$ perpendicularly to the slit, so that their wave vectors expressed in the spherical coordinates $\kappa, \theta$ with $\varphi=0$ are given by

$\boldsymbol{\kappa} \equiv \boldsymbol{\kappa}(\theta)=\{\kappa \sin \theta, 0, \kappa \cos \theta\}$.

In addition, we assume that the incident neutron beam is directed at the Bragg angle $\theta_{\mathrm{B}}$ with respect to reflecting planes and has Gaussian spreading over the

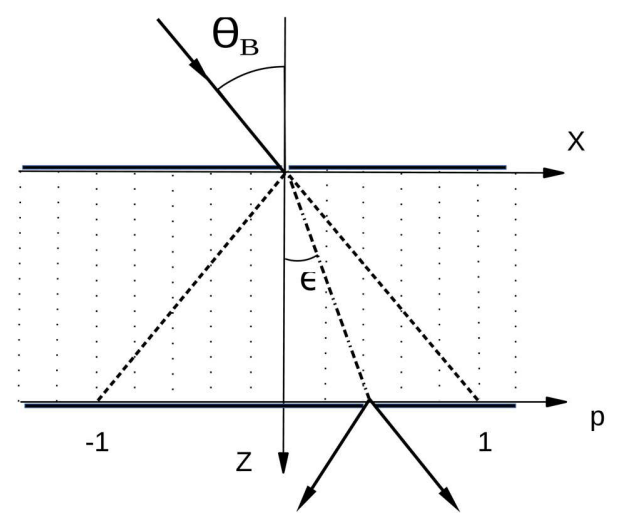

Fig. 1. Scheme of the symmetric Laue diffraction of a spherical neutron wave

angle $\theta$. Then the wave function (1) may be rewritten as

$\Psi_{\mathrm{in}}(\mathbf{r}, t)=\int_{0}^{\infty} G_{e}(E) \Psi_{E}^{\mathrm{in}}(\mathbf{r}) e^{-i E t / \hbar} d E$,

where $G_{e}(E)$ characterizes the energy distributions of incident neutrons, and the function $\Psi_{E}^{\text {in }}(\mathbf{r})$, which describes neutrons with fixed energy $E$, is given by

$\Psi_{E}^{\mathrm{in}}(\mathbf{r})=\int d \theta G_{a}(\theta) e^{i \kappa \mathbf{r}}$

Let the angular distribution be

$G_{a}(\theta)=\frac{1}{(\sqrt{2 \pi} \sigma)^{1 / 2}} \exp \left\{-\frac{(\Delta \theta)^{2}}{4 \sigma^{2}}\right\}$

where

$\Delta \theta=\theta_{\mathrm{B}}-\theta$

is a deviation from the Bragg angle $\theta_{\mathrm{B}}$,

$\sigma^{2}=\left\langle(\Delta \theta)^{2}\right\rangle$

determines a mean-square deviation of $\theta$ from $\theta_{\mathrm{B}}$. Let $\sigma$ be much less than unity. This enables us to spread the integration limits over $\theta$ from $-\infty$ to $\infty$. At the same time, we suppose that $\sigma$ exceeds significantly the angular range, where the diffraction proceeds. This assumption allows us to use the sphericalwave approximation introduced by Kato, i.e., to replace the distribution $G_{a}(\theta)$ by a constant. We put it equal to unity. 
We shall consider the neutron wave scattering by atomic nuclei of the crystal. For simplicity, we do not touch upon the magnetic crystals, in which the neutron scattering by electrons is also significant [19]. In addition, the nuclei are assumed to be unpolarized. All this allows us to omit the neutron spin wave function [19]. Then the coherent scattering of neutrons by a single elementary cell of the crystal from the state with the wave vector $\boldsymbol{\kappa}$ to $\boldsymbol{\kappa}^{\prime}$ is determined by the amplitude

$F\left(\boldsymbol{\kappa}, \boldsymbol{\kappa}^{\prime}\right)=\sum_{j} e^{i \mathbf{Q} \boldsymbol{\rho}_{j}} \bar{f}_{j}\left(\boldsymbol{\kappa}, \boldsymbol{\kappa}^{\prime}\right)$,

where $\mathbf{Q}=\boldsymbol{\kappa}-\boldsymbol{\kappa}^{\prime}$ is the scattering vector, the radiusvector $\boldsymbol{\rho}_{j}$ defines the position of the $j$ th atom within the elementary cell, $\bar{f}_{j}\left(\boldsymbol{\kappa}, \boldsymbol{\kappa}^{\prime}\right)$ is the coherent scattering amplitude of low-energy neutrons by the $j$ th nucleus:

$\bar{f}_{j}\left(\boldsymbol{\kappa}, \boldsymbol{\kappa}^{\prime}\right)=-\bar{a}_{j} e^{-W_{j}(\mathbf{Q})}+\bar{f}_{j}^{\mathrm{res}}\left(\boldsymbol{\kappa}, \boldsymbol{\kappa}^{\prime}\right)$,

where $\bar{a}_{j}$ is the coherent scattering length of neutrons by the $j$ th nucleus, $e^{-W_{j}(\mathbf{Q})}$ is the square root of the Debye-Waller factor, $\bar{f}_{j}^{\text {res }}\left(\boldsymbol{\kappa}, \boldsymbol{\kappa}^{\prime}\right)$ is the coherent resonant scattering amplitude. In a vicinity of the isolated resonance, it is given by

$$
\begin{aligned}
& \bar{f}_{j}^{\mathrm{res}}\left(\boldsymbol{\kappa}, \boldsymbol{\kappa}^{\prime}\right)=-c_{j}\left(\frac{2 I_{e}+1}{2 I_{g}+1}\right) \frac{\Gamma_{n}}{2 \kappa} \times \\
& \times \sum_{\left\{n_{s}^{\prime}\right\}}\left\langle\frac{\left(\exp \left[-i \boldsymbol{\kappa}^{\prime} \mathbf{u}_{j}\right]\right)_{\left\{n_{s}^{0}\right\}\left\{n_{s}^{\prime}\right\}}\left(\exp \left[i \boldsymbol{\kappa} \mathbf{u}_{j}\right]\right)_{\left\{n_{s}^{\prime}\right\}\left\{n_{s}^{0}\right\}}}{E-E_{0}-\sum_{s} \hbar \omega_{s}\left(n_{s}^{\prime}-n_{s}^{0}\right)+i \frac{\Gamma}{2}}\right\rangle,
\end{aligned}
$$

where $c_{j}$ is the probability of finding the resonant isotope in the $j$ th site, $I_{g}$ and $I_{e}$ are the nuclear spins in the ground and excited compound states, respectively, $E_{0}$ and $\Gamma$ are the energy and width of the resonant level, $\Gamma_{n}$ is the partial neutron width, $\mathbf{u}_{j}$ is the displacement of the $j$ th nucleus from the equilibrium position, $\left\{n_{s}^{0}\right\}$ and $\left\{n_{s}^{\prime}\right\}$ are sets of phonon numbers in the initial and final states of the crystal, $\omega_{s}$ are the phonon frequencies, and the brackets $\langle\ldots\rangle$ denote averaging over the initial states of the crystal lattice. The sum in Eq. (10) can be transformed to the integral:

$\sum_{\left\{n_{s}^{\prime}\right\}}\langle\ldots\rangle=-i e^{-W_{j}(\boldsymbol{\kappa})} e^{-W_{j}\left(\boldsymbol{\kappa}^{\prime}\right)} \times$ $\times \int_{0}^{\infty} \frac{d t}{\hbar} e^{i\left(E-E_{0}\right) t / \hbar-\Gamma t / 2 \hbar+\varphi_{j}(t)}$,

where

$\varphi_{j}(t)=\sum_{s} \frac{\hbar}{2 M_{j} N \omega_{s}} \times$

$\times\left[y_{j s} \bar{n}_{s} e^{i \omega_{s} t}+y_{j s}^{*}\left(\bar{n}_{s}+1\right) e^{-i \omega_{s} t}\right]$,

$M_{j}$ is the mass of the $j$ th atom, $N$ is the number of elementary cells, $\bar{n}_{s}$ is the average number of phonons of the $s$ th normal vibration with polarization $\mathbf{v}_{j s}$, and $y_{j s}=\left(\boldsymbol{\kappa} \mathbf{v}_{j s}\right)\left(\boldsymbol{\kappa}^{\prime} \mathbf{v}_{j s}^{*}\right)$.

In the framework of the Debye model of a crystal with one atom per the elementary cell, by ignoring the anisotropy of vibrations, we can rewrite this expression as (see also [20])

$\varphi_{j}(t)=\frac{3}{2} \frac{\left(\mathbf{p} \cdot \mathbf{p}^{\prime}\right)}{M\left(k_{\mathrm{B}} \Theta_{\mathrm{D}}\right)^{3}} \int_{0}^{\omega_{\max }} \hbar^{2} \omega d \omega \times$
$\times\left[\bar{n}_{\omega} e^{i \omega t}+\left(\bar{n}_{\omega}+1\right) e^{-i \omega t}\right]$,

where $\mathbf{p}=\hbar \boldsymbol{\kappa}$ and $\mathbf{p}^{\prime}=\hbar \boldsymbol{\kappa}^{\prime}$ are the initial and final momenta of neutrons, $\Theta_{\mathrm{D}}$ is the Debye temperature, and $\omega_{\max }=k_{\mathrm{B}} \Theta_{\mathrm{D}} / \hbar$ is the maximal frequency of phonons.

In the case of fast collisions, when $\hbar \omega_{\max } / \Gamma \ll 1$, the expression (11) reduces to

$\sum_{\left\{n_{s}^{\prime}\right\}}\langle\ldots\rangle=\frac{e^{-W_{j}(\mathbf{Q})}}{E-E_{0}+i \frac{\Gamma}{2}}$.

\section{The Wave Function}

According to collision theory [21], every plane wave $e^{i \kappa \mathbf{r}}$ of the wave packet (1) is scattered independently of one another, giving rise to the wave function $\boldsymbol{\psi}_{\boldsymbol{\kappa}}(\mathbf{r})$. Respectively, the wave function of the neutron born by the incident wave packet (4) in the approximation $G_{a}(\theta) \approx 1$ takes the form

$\Psi_{E}(\mathbf{r})=\int_{-\infty}^{\infty} d \theta \boldsymbol{\psi}_{\boldsymbol{\kappa}(\theta)}(\mathbf{r})$,

where the functions $\psi_{\boldsymbol{\kappa}(\theta)}(\mathbf{r})$ are generated by the plane waves $e^{i \kappa(\theta) \mathbf{r}}$. In the two-wave case, the wave vectors of the refracted and diffracted waves inside the crystal are, respectively, $\mathbf{k}(\theta)$ and $\mathbf{k}_{1}(\theta)=\mathbf{k}(\theta)+$ $+\mathbf{h}_{1}$, where $\mathbf{h}_{1}$ denotes a reciprocal lattice vector.

ISSN 2071-0194. Ukr. J. Phys. 2018. Vol. 63, No. 2 
The components of the vectors $\mathbf{k}(\theta)$ and $\boldsymbol{\kappa}(\theta)$ along the face surface $z=0$ coincide. Therefore, the vector $\mathbf{k}_{\nu}(\theta)$ can be written as

$\mathbf{k}_{\nu}(\theta)=\boldsymbol{\kappa}_{\nu}(\theta)+\delta(\theta) \mathbf{n}, \quad \boldsymbol{\kappa}_{1}(\theta)=\boldsymbol{\kappa}_{0}(\theta)+\mathbf{h}_{1}$,

where $\mathbf{n}$ is the unit vector along the axis $z$.

Then the wave function $\Psi_{E}(\mathbf{r})$ at $0<z<D$, where $D$ is the crystal thickness, transforms to

$$
\begin{aligned}
& \Psi_{E}(\mathbf{r})=\sum_{\nu=0,1} \Psi_{E}^{(\nu)}(\mathbf{r}), \\
& \Psi_{E}^{(\nu)}(\mathbf{r})=\int_{-\infty}^{\infty} d \theta \psi_{\boldsymbol{\kappa}_{\nu}(\theta)}(\mathbf{r}),
\end{aligned}
$$

with $\psi_{\boldsymbol{\kappa}_{\nu}(\theta)}(\mathbf{r})$ yielded by

$\psi_{\boldsymbol{\kappa}_{\nu}(\theta)}(\mathbf{r})=\sum_{\iota=1,2} C_{\nu}^{(\iota)}(\theta) e^{i \boldsymbol{\kappa}_{\nu}(\theta) \mathbf{r}+i \delta_{\iota}(\theta) z}$

The amplitudes $C$ and the wave vectors $\mathbf{k}$ in the two-wave case are determined by the algebraic equations $[2]$

$$
\begin{aligned}
& \left(k^{2}(\theta) / \kappa^{2}(\theta)-1\right) C_{0}=g_{00} C_{0}+g_{01} C_{1}, \\
& \left(k_{1}^{2}(\theta) / \kappa^{2}(\theta)-1\right) C_{1}=g_{10} C_{0}+g_{11} C_{1},
\end{aligned}
$$

where the dimensionless scattering matrix

$g_{\mu \nu}=\frac{4 \pi}{\kappa^{2} v_{0}} F\left(\boldsymbol{\kappa}_{\nu}, \boldsymbol{\kappa}_{\mu}\right), \quad \mu, \nu=0,1$.

Here, $v_{0}$ stands for the volume of the elementary cell, the wave vectors $\boldsymbol{\kappa}_{0}=\boldsymbol{\kappa}\left(\theta_{\mathrm{B}}\right)$ and $\boldsymbol{\kappa}_{1}=\boldsymbol{\kappa}_{0}+\mathbf{h}_{1}$.

The similar solutions of Eqs. (19) given in [2] depend on the angle

$\alpha=\frac{2 \boldsymbol{\kappa} \mathbf{h}_{1}+\mathbf{h}_{1}^{2}}{\kappa^{2}}$,

to be equal zero at the Bragg resonance. This angle is related to $\Delta \theta$ by [5]

$\alpha=2 \sin \left(2 \theta_{\mathrm{B}}\right) \Delta \theta$.

It is convenient to introduce the deviation parameter

$\eta=\frac{1}{2 \sqrt{g_{01} g_{10}}}\left(\alpha-\alpha_{0}\right)$,

where the angular shift

$\alpha_{0}=g_{11}-g_{00}$.
Then, for the parameter $\delta_{\iota}(\theta)$, we find the expression

$\delta_{\iota}(\theta)=\frac{\kappa g_{00}}{2 \gamma_{0}}-\frac{\pi}{\Lambda_{L}}\left(\eta+(-1)^{\iota+1} \sqrt{1+\eta^{2}}\right)$,

where

$\gamma_{0}=\cos \theta_{\mathrm{B}}$

and

$\Lambda_{L}=\frac{2 \pi \gamma_{0}}{\kappa \sqrt{g_{01} g_{10}}}$

means the Pendellösung distance in the case of weakly absorbing crystals, when Bragg's condition $\eta=0$ is exactly fulfilled (see also [7]).

The amplitudes of the waves in the symmetric geometry are

$C_{0}^{(\iota)}(\eta)=\frac{1}{2}\left(1+(-1)^{\iota} \frac{\eta}{\sqrt{1+\eta^{2}}}\right)$
$C_{1}^{(\iota)}(\eta)=\frac{(-1)^{\iota}}{2}\left(\frac{g_{10}}{g_{01}}\right)^{1 / 2} \frac{1}{\sqrt{1+\eta^{2}}}$.

The distribution of the neutron intensity over the base of the Borrmann triangle is analyzed with the aid of the scanning slit located on the rear surface and also directed along the axis $y$. The position of such a slit is determined by the reduced coordinate [6]

$p=\tan \epsilon / \tan \theta_{\mathrm{B}}$

where $\tan \epsilon=x_{s} / D$, and $x_{s}$ is the usual coordinate of the scanning slit.

In addition, we expand the exponents of $e^{i \boldsymbol{\kappa}_{\nu}(\theta) \mathbf{r}}$ in $\Delta \theta$. Keeping the linear terms and using relation (22), we get

$e^{i \boldsymbol{\kappa}_{\nu}(\theta) \mathbf{r}} \approx \exp \left\{i \frac{\kappa z}{4 \gamma_{0}}(1-p) \alpha\right\} e^{i \boldsymbol{\kappa}_{\nu} \mathbf{r}}$,

where $\boldsymbol{\kappa}_{\nu}=\boldsymbol{\kappa}_{\nu}\left(\theta_{\mathrm{B}}\right)$. Inserting here (23), one gets finally

$e^{i \boldsymbol{\kappa}_{\nu}(\theta) \mathbf{r}} \approx B(p ; z) \exp \left\{i \frac{\pi z}{\Lambda_{L}}(1-p) \eta\right\} e^{i \boldsymbol{\kappa}_{\nu} \mathbf{r}}$,

where we introduced the designation

$B(p ; z)=\exp \left\{i \frac{\kappa z}{4 \gamma_{0}}(1-p) \alpha_{0}\right\}$.

In the case of isotropic vibrations, when the angle $\alpha_{0}=0$, the function $B(p ; z)=1$. 
By substitution (18), (25), (28), and (31) into Eq. (17), we find the integral representation for the wave function behind the crystal:

$$
\begin{aligned}
& \Psi_{E}^{(\nu)}(\mathbf{r})=B(p ; D) \frac{\sqrt{g_{01} g_{10}}}{\sin 2 \theta_{\mathrm{B}}} \exp \left\{i \frac{\kappa g_{00} D}{2 \gamma_{0}}\right\} \times \\
& \times \int_{-\infty}^{\infty} d \eta \sum_{\iota=1,2} C_{\nu}^{(\iota)}(\eta) e^{N S_{\iota}(\eta)} e^{i \boldsymbol{\kappa}_{\nu} \mathbf{r}},
\end{aligned}
$$

where

$N=\frac{\pi D}{\left|\Lambda_{L}\right|}$

and

$S_{\iota}(\eta)=-i\left(\left|\Lambda_{L}\right| / \Lambda_{L}\right)\left[p \eta+(1)^{\iota+1} \sqrt{1+\eta^{2}}\right]$.

For a crystal, whose thickness $D \gg\left|\Lambda_{L}\right| / \pi$, the large number $N$ allows us to estimate the integral over $\eta$ with the aid of the saddle-point method (see, e.g., [22]). By equating the derivative $S_{\iota}^{\prime}(\eta)$ to zero, we find the saddle point for the $\iota$ th wave:

$\eta_{0}^{(\iota)}=(-1)^{\iota} \frac{p}{\sqrt{1-p^{2}}}$.

Then the integration contour is displaced to the complex plane $\eta=\eta_{r}+i \eta_{i}$, where $\eta_{r}$ and $\eta_{i}$ represent the real and imaginary parts of $\eta$. This contour crosses the $\iota$ th saddle point along the line, which indicates a steepest decent of the function $S_{\iota}(\eta)$. Along such a line, $\operatorname{Im} S_{\iota}(\eta)=$ const, and the function $\operatorname{Re} S_{\iota}(\eta)$ is maximal at the point $\eta_{0}$. The line is declined with respect to the real axis $\eta_{r}$ at the angle

$\vartheta_{\iota}= \pm \frac{\pi}{2}-\frac{1}{2} \arg S_{\iota}^{\prime \prime}\left(\eta_{0}\right)$,

where the second derivative of $S_{\iota}(\eta)$ at the saddle point equals

$S_{\iota}^{\prime \prime}\left(\eta_{0}\right)=i(-1)^{\iota}\left(\frac{\left|\Lambda_{L}\right|}{\Lambda_{L}}\right)\left(1-p^{2}\right)^{3 / 2}$.

Inserting (38) into (37), we get that, along the base of the Borrmann triangle at $|p|<1$,

$\vartheta_{\iota}=(-1)^{\iota} \frac{\pi}{4}+\arg \sqrt{\Lambda_{L}}$.

Evaluating integral (33) with the aid of the saddlepoint method (see, e.g., [22]), we have

$\Psi_{E}^{(\nu)}(\mathbf{r})=B(p ; D) \exp \left(i \frac{\kappa g_{00} D}{2 \gamma_{0}}\right) \times$ $\times \sum_{\iota=1,2} C_{\nu)}^{(\iota)} e^{N S_{\iota}\left(\eta_{0}\right)} \sqrt{\frac{2 \pi}{N\left|S_{\iota}^{\prime \prime}\left(\eta_{0}\right)\right|}} e^{i \vartheta_{\iota}} e^{i \boldsymbol{\kappa}_{\nu)} \mathbf{r}}$,

where the amplitudes $C_{\nu}^{(\iota)}=C_{\nu}^{(\iota)}\left(\eta_{0}^{\iota}\right)$ at the saddle points are

$C_{0}^{(\iota)}=\frac{1}{2}(1+p)$

$C_{1}^{(\iota)}=\frac{(-1)^{\iota}}{2}\left(\frac{g_{10}}{g_{01}}\right)^{1 / 2} \sqrt{1-p^{2}}$.

Then, substituting (38), (39), and (41) into (40), we get the wave function of neutrons transmitted through the crystal $(z>D)$. We found that the refracted neutrons are described by

$\Psi_{E}^{(0)}(p ; \mathbf{r})=\frac{1}{2} \frac{\mathcal{A}_{0}(p)}{\left(1-p^{2}\right)^{1 / 4}}\left(\frac{1+p}{1-p}\right)^{1 / 2} \times$
$\times \exp \left(i \frac{\kappa g_{00} D}{2 \gamma_{0}}\right) \sqrt{\frac{2 \Lambda_{L}}{D}}\left[e^{i z(p)}+e^{-i z(p)}\right] e^{i \boldsymbol{\kappa}_{0} \mathbf{r}}$

and the diffracted ones by

$\Psi_{E}^{(1)}(p ; \mathbf{r})=\frac{1}{2} \frac{\mathcal{A}_{1}(p)}{\left(1-p^{2}\right)^{1 / 4}} \times$

$\times \exp \left(i \frac{\kappa g_{00} D}{2 \gamma_{0}}\right) \sqrt{\frac{2 \Lambda_{L}}{D}}\left[e^{i z(p)}-e^{-i z(p)}\right] e^{i \boldsymbol{\kappa}_{1} \mathbf{r}}$,

where

$z(p)=\frac{\pi D}{\Lambda_{L}} \sqrt{1-p^{2}}+\frac{\pi}{4}$

and

$\mathcal{A}_{0}(p)=B(p ; D) \frac{\sqrt{g_{01} g_{10}}}{\sin 2 \theta_{\mathrm{B}}}$,

$\mathcal{A}_{1}(p)=\left(\frac{g_{10}}{g_{01}}\right)^{1 / 2} \mathcal{A}_{0}(p)$

The intensities of the monochromatic neutron beams are determined by

$I_{E}^{(\nu)}(p)=\left|\Psi_{E}^{(\nu)}(p ; \mathbf{r})\right|^{2}$.

For the refracted beam, we have

$I_{E}^{(0)}(p)=\frac{\left|\mathcal{A}_{0}(p)\right|^{2}}{\sqrt{1-p^{2}}} \sqrt{\left|\frac{1+p}{1-p}\right|} \frac{2\left|\Lambda_{L}\right|}{D} e^{-\mu D / \gamma_{0}} \times$
$\times\left[\sinh ^{2}\left(\frac{\pi D}{\sigma_{L}} \sqrt{1-p^{2}}\right)+\cos ^{2}\left(\frac{\pi D}{\tau_{L}} \sqrt{1-p^{2}}+\frac{\pi}{4}\right)\right]$,

ISSN 2071-0194. Ukr. J. Phys. 2018. Vol. 63, No. 2 
while, for the diffracted beam,

$$
\begin{aligned}
& I_{E}^{(1)}(p)=\frac{\left|\mathcal{A}_{1}(p)\right|^{2}}{\sqrt{1-p^{2}}} \frac{2\left|\Lambda_{L}\right|}{D} e^{-\mu D / \gamma_{0}} \times \\
& \times\left[\sinh ^{2}\left(\frac{\pi D}{\sigma_{L}} \sqrt{1-p^{2}}\right)+\cos ^{2}\left(\frac{\pi D}{\tau_{L}} \sqrt{1-p^{2}}-\frac{\pi}{4}\right)\right] .
\end{aligned}
$$

Here, we introduced the notation

$\frac{1}{\Lambda_{L}}=\frac{1}{\tau_{L}}+i \frac{1}{\sigma_{L}}$

and the absorption coefficient far from the Bragg condition

$\mu=\kappa \operatorname{Im} g_{00}=\sigma_{a} / v_{0}$,

$\sigma_{a}=\frac{4 \pi}{\kappa} \operatorname{Im} F(\boldsymbol{\kappa}, \boldsymbol{\kappa})$ is the absorption cross-section of neutrons by an elementary cell.

The total intensities of the beams are obtained by the averaging of (47) and (48) with the weight $\left|G_{e}(E)\right|^{2}$,

$I^{(\nu)}(p)=\int_{0}^{\infty} d E\left|G_{e}(E)\right|^{2} I_{E}^{(\nu)}(p)$.

\section{Conclusion}

So, we have analyzed the symmetric Laue diffraction of collimated neutron beams in perfect crystals containing nuclei with low-lying resonant levels. In the spherical-wave approximation of Kato, making estimations by the saddle-point method, we have derived Eqs. (42) and (43) for the wave functions of the refracted and diffracted neutrons, as well as Eqs. (47) and (48) for their intensities. The vicinity of the point $|p|=1$ should be excluded in these formulas, since, at the point $|p|=1$, the second derivative $S_{\iota}^{\prime \prime}\left(\eta_{0}^{\iota}\right)=0$. In this case, the main idea of the saddlepoint method that the function $\operatorname{Re}\left\{N S_{\iota}(\eta)\right\}$ is represented by a sharp peak along the line of the steepest decent fails. Only such quick fall-down enables one to apply the Laplace method of approximate integration [22].

Our equations (42) and (43) for the wave function of neutrons differ formally from those for x-rays [7], only by the additional factor $B(p ; z)$. It depends on the angular shift $\alpha_{0}=g_{00}-g_{11}$, which vanishes only in the case of isotropic vibrations of the crystal lattice. Only then $B(p ; z)=1$. Another significant difference of our formulas from those describing

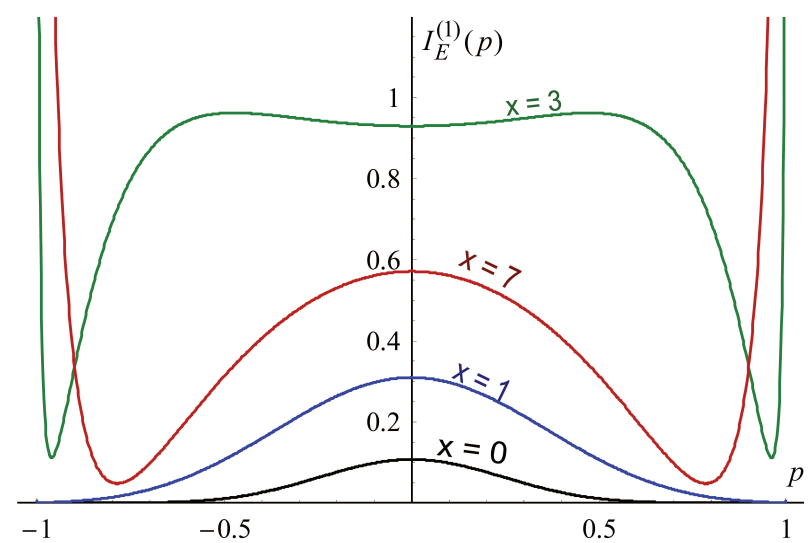

Fig. 2. Distribution of the intensity of the diffracted beam $I_{E}^{(1)}(p)$ over the base of the Borrmann triangle for $\mu_{\mathrm{res}} D=20$

the x-ray diffraction [7] is that the coherent scattering amplitudes of neutrons contain the resonant amplitude $\bar{f}_{j}^{\text {res }}\left(\boldsymbol{\kappa}, \boldsymbol{\kappa}^{\prime}\right)$, whose imaginary part in a vicinity of the resonance is comparable with its imaginary part. As a result, the anomalous absorption of waves in both cases has different character (for details, see $[1,2])$.

In order to illustrate the role of the resonant scattering, we have done numerical calculations in a simplified case where the isotropic crystal lattice contains one atom per unit cell, $B(p ; z)=1$, and the potential scattering can be neglected compared to the resonant one. The role of phonons is taken into account by the factor $e^{-W(Q)}$ of Eq. (14). For definiteness, we took $e^{-2 W(Q)}=0.8$. In this approximation, the absorption coefficient depending on the resonance detuning $x=2\left(E-E_{0}\right) / \Gamma$ can be written as

$\mu(x)=\frac{\mu_{\mathrm{res}}}{1+x^{2}}$,

where $\mu_{\text {res }}$ is the resonant value with $\kappa \approx \kappa_{0}$. At the same time, the function (44) becomes

$z(p)=-\frac{\mu_{\mathrm{res}} D}{2 \gamma_{0}} \frac{e^{-W(Q)}}{(x+i)} \sqrt{1-p^{2}}+\frac{\pi}{4}$.

The intensities of the diffracted and refracted beams, calculated in units of $e^{-\mu_{\text {res }} D / \gamma_{0}}$, as functions of the parameter $p$ for different values of detuning of the resonance $x$, are drawn in Figs. 2 and 3 .

The neutrons pass the crystal mainly within the Borrmann triangle. Outside it, the functions $I_{E}^{(\nu)}(p)$ rapidly fall down. We see here that the curves $I_{E}^{(1)}(p)$ 


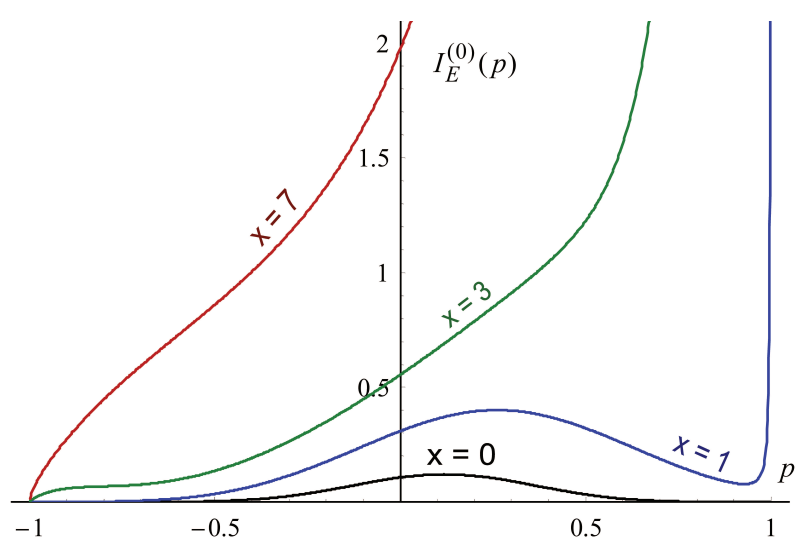

Fig. 3. Distribution of the intensity of the refracted beam $I_{E}^{(0)}(p)$ over the base of the Borrmann triangle for $\mu_{\mathrm{res}} D=20$

for the diffracted neutrons are symmetric with respect to the reflection $p \rightarrow-p$. Far from the resonance, $(x \gg 1)$, the intensity of the diffracted neutrons symmetrically increases near the margins of the Borrmann triangle, while the curves $I_{E}^{(0)}(p)$, which describe the refracted beams, rapidly grow up near the point $p=1$ corresponding to direct passage of neutrons through the crystal. In a close vicinity to the resonance, $(x \approx 0)$, the intensities $I_{E}^{(\nu)}(p)$ tend to be concentrated at the center of the triangle, which is similar to the situation with the x-ray diffraction [7]. Thus, we see the same effect as in the case of x-rays. Namely, the energy in strongly absorbing crystals floats along the reflecting planes (see also [6]. Equations (47) and (48) contain both the smooth term $\sinh ^{2}\left[\left(\pi D / \sigma_{L}\right) \sqrt{1-p^{2}}\right]$ and the oscillating one $\cos ^{2}\left[\left(\pi D / \tau_{L}\right) \sqrt{1-p^{2}} \pm \pi / 4\right]$. With a deviation from the resonance, the last term begins to manifest itself. Specifically, the diffracted beam intensity $I_{E}^{(1)}(p)$ at $x=3$ is higher than that at $x=7$. In addition, the curve $I_{E}^{(0)}(p)$ at $x=1$ attributes a large curvature in a vicinity of $p=1$ also due to the oscillating term.

1. A.M. Afanas'ev, Yu. Kagan. Suppression of inelastic channels in resonant nuclear scattering in crystals. Zh. Exp. Teor. Fiz. 48, 327 (1965) [Sov. Phys. JETP 21, 215 (1965)].

2. Yu. Kagan, A.M. Afanas'ev. Suppression of inelastic channels in resonance scattering of neutrons in regular crystals. Zh. Exp. Teor. Fiz. 49, 1504 (1965) [Sov. Phys. JETP 22, 1032 (1966)].
3. S.Sh. Shil'shtein et al. Anomalous transmission of neutrons in a perfect CdS crystal. Pis'ma ZhETF 12, 80 (1970) [JETP Lett. 12, 56 (1970)].

4. S.Sh. Shil'shtein, V.A. Somenkov, V.P. Dokashenko. Suppression of $(n, \gamma)$ reaction in resonant scattering of neutrons by a perfect CdS crystal. Pis'ma ZhETF 13, 301 (1971) [JETP Lett. 13, 214 (1971)].

5. W.H. Zachariasen. Theory of X-ray Diffraction in Crystals (Wiley, 1945).

6. B.W. Batterman, H. Cole. Dynamical diffraction of X-rays by perfect crystals. Rev. Mod. Phys. 36, 681 (1964).

7. A. Authier. Dynamical Theory of X-ray Diffraction (Oxford Univ. Press, 2001).

8. Z.G. Pinsker. Dynamical Scattering of X-Rays in Crystals (Nauka, 1982; Springer, 1978).

9. N. Kato. The energy flow of X-rays in an ideally perfect crystal: comparison between theory and experiments. Acta Cryst. 13, 349 (1960).

10. N. Kato. A theoretical study of Pendellösung fringes. I. General considerations. Acta Cryst. 14, 526 (1961).

11. N. Kato. A theoretical study of Pendellösung fringes. II. Detailed discussion based upon a spherical wave theory. Acta Cryst. 14, 627 (1961).

12. N. Kato. Pendellösung fringes in distorted crystals III. Application to homogeneously bent crystals. J. Phys. Soc. Japan 19, 971 (1964).

13. C.G. Shull. Observation of Pendellösung fringe structure in neutron diffraction. Phys. Rev. Lett. 21, 1585 (1968).

14. C.G. Shull. Perfect crystals and imperfect neutrons. J. Appl. Phys. 6, 257 (1973).

15. C.G. Shull, W.M. Shaw. Neutron Pendellösung fringe structure in the Laue diffraction by germanium. Z. Naturforsch. 28a, 657 (1973).

16. E.O. Vezhlev et al. Effect of anomalous absorption of neutrons undergoing Laue diffraction at Bragg angles close to $\pi / 2$. Pis'ma ZhETF 96, 3 (2012) [JETP Lett. 96, 1 (2012)].

17. V.V. Voronin et al. Analysis of spatial resolution of an experiment on verification of the equivalence principle for a neutron by the diffraction method. Pis'ma ZhTF 43, 75 (2017) [Technical Phys. Lett. 43, 270 (2017)].

18. A.Ya. Dzyublik, V.Yu. Spivak. Laue diffraction of spherical Mössbauer waves. Ukr. J. Phys. 61, 826 (2016).

19. I.I. Gurevich, L.V. Tarasov. Low energy neutron phisics (Nauka, 1965; North-Holland, 1968).

20. A. Akhiezer, I. Pomeranchuk. Some Problems of Nuclear Theory (GITTL, 1950) (in Russian).

21. M.L. Goldberger, K.M. Watson. Collision Theory (Wiley, 1964).

22. M.A. Lavrentiev, B.V. Shabat, Methods of the Theory of Functions of Complex Variable (Nauka, 1973) (in Russian).

Received 10.11.17

ISSN 2071-0194. Ukr. J. Phys. 2018. Vol. 63, No. 2 
О.Я. Дзюблик, B.I. Слісенко, В.В. Михайловсъкий

СИМЕТРИЧНА ДИФРАКЦІЯ ЛАУЕ

СФЕРИЧНИХ НЕЙТРОННИХ ХВИЛЬ

У ПОГЛИНАЮЧИХ КРИСТАЛАХ

$\mathrm{P}$ е $з$ ю м е

Відому теорію Като дифракції Лауе сферичних рентгенівських хвиль узагальнено на випадок дифракції нейтронів у сильно поглинаючих кристалах, беручи до уваги як потенціальне, так і резонансне розсіяння нейтронів ядрами. Для оцінки кутових інтегралів застосовується метод перевалу, який є більш адекватний у випадку сильно поглинаючих кристалів, ніж наближення стаціонарної фази, що використовувалося Като. Встановлено, що розподіл інтенсивності дифрагованих і заломлених пучків вздовж основи трикутника Бормана сильно залежить від відхилення енергії нейтронів від ядерного резонансного рівня. 